\title{
HIGHLIGHTS
}

RHEUMATOID ARTHRITIS

\section{Synergistic effects of growth factors drive an RA phenotype in fibroblast-like synoviocytes}

The interplay between growth factors and cytokines is an important aspect of the complex microenvironment of chronically inflamed rheumatoid synovia; however, these interactions are still poorly understood as a result of the limited ability of animal models to reflect human disease. Evidence from in vitro studies by Sanna Rosengren et al. suggests that transforming growth factor $\beta$ (TGF- $\beta$ ) and platelet-derived growth factor (PDGF) act synergistically to direct synovial fibroblasts toward a more aggressive phenotype in response to tumor necrosis factor (TNF). "We chose cytokines such as TNF because they are established targets for the treatment of rheumatoid arthritis, and combined them with growth factors due to their presence in rheumatoid synovia," explains David Boyle, the study's lead investigator.

The researchers noted that a combination of TGF- $\beta$ and PDGF (termed 2GF) augmented production of interleukin- 6 and matrix metallaproteinase-3 RNA and protein by fibroblast-like synoviocytes in response to TNF treatment. Furthermore, the potentiating role of $2 \mathrm{GF}$ was observed even when added to the cells up to $6 \mathrm{~h}$ prior to stimulation with TNF. The investigators also found a considerable increase in c-Jun $\mathrm{N}$-terminal kinase (JNK) phosphorylation following 15 min of combined treatment with TNF and $2 \mathrm{GF}$, suggesting that $2 \mathrm{GF}$ signals through a JNK-dependent pathway. Indeed, further experiments using specific pathway inhibitors identified the phosphoinositide 3-kinase (PI3K) pathway as the most likely candidate for the activity of $2 \mathrm{GF}$.

Interestingly, Boyle's research team also found that the 2GF-mediated increase in proinflammatory cytokine levels in fibroblast-like synoviocytes could be inhibited by treatment with imatinib mesylate-a receptor tyrosine kinase inhibitor currently being tested as a therapy in rheumatoid arthritis (RA) under the name Gleevec ${ }^{\circledR}$.

The authors suggest that TGF- $\beta$ and PDGF direct the response of synovial cells toward an RA phenotype, and comment that their findings further highlight the therapeutic potential for targeting the PI3K pathway. "Targeting growth factor signaling may be an indirect method to reduce cytokine and protease production in RA synovitis while minimizing the impact on host defense," concludes Boyle.

Rowan Higgs

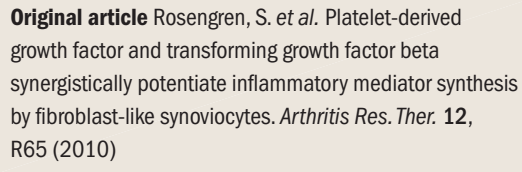

